\title{
EXPERIENCIAS DE INCORPORACIÓN DE APLICACIONES SEMÁNTICAS A LA EDUCACIÓN
}

\section{INCORPORATING SEMANTIC APPLICATIONS IN EDUCATION, THE STORY SO FAR}

\author{
Ma del Mar Sánchez Vera; mmarsanchez@um.es \\ Ma Paz Prendes Espinosa; pazprend@um.es
}

Francisco Martínez Sánchez; pacomar@um.es

Universidad de Murcia (España)

Patrick Carmichael; w.p.carmichael@ljmu.ac.uk

Agustina Martinez-Garcia; a.martinez-garcia@ljmu.ac.uk

Universidad Liverpool John Moores (Inglaterra)

\section{RESUMEN}

La Web 3.0 (Web Semántica) es una realidad en la red y proporciona numerosas oportunidades para mejorar y apoyar la enseñanza y el aprendizaje. La mayoría de sus aplicaciones actuales se centran en áreas como la construcción de conocimiento y entornos personales de aprendizaje, habiendo muy pocos ejemplos de aplicación en areas en dónde la adquisición de conocimiento es un proceso complejo y cambia rápidamente, como por ejemplo en entornos de aprendizaje basado en casos. Este articulo recopila algunas de las experiencias más relevantes de incorporación de tecnologías semánticas en este tipo de entornos educativos, llevadas a cabo por los grupos de investigación GITE y el proyecto Ensemble.

PALABRAS CLAVE: Web Semántica, aplicaciones visuales, recursos educativos, universidad, aprendizaje basado en casos

\section{ABSTRACT}

Web 3.0 and its associated technologies have the potential to support and enhance teaching and learning environments. Whilst there are some applications of the Semantic Web in Education, highlighting areas such as resource discovery or personal networking or educational administration, there is a lack of applications in areas where knowledge is 
complex, changing or contested, as in case-based learning environments. This article describes some of the experiences of introducing semantic technologies into these types of learning environments that have been carried out by the GITE Research Group and the Ensemble Project team in collaboration with teachers and students across various educational settings.

KEYWORDS: Semantic Web, visualisation tools, higher education, learning resources, casebased learning

\section{INTRODUCCIÓN: ¿QUÉ ES LA WEB SEMÁNTICA?}

Existen varias definiciones de la Web Semántica: Web 3.0, Web extendida o Web ontológica. Sea cual sea la definición escogida todas hacen referencia a una Web que no sólo se limita a proporcionar acceso a información diversa sino que dota de significado a esa información de forma que los ordenadores pueden interpretarla y procesarla. La Web original o Web 1.0 es una web sintáctica, nos movemos por la información a través de vínculos o enlaces y desde el punto de vista de la tecnología, sólo sabemos que una información determinada está relacionada con otra pero no conocemos de qué forma, mientras que la Web Semántica trata de construir una base de conocimiento que nos posibilite acceder a la información de una manera más comprensiva. La Web 2.0, más conocida como 'web social' representa un punto intermedio entre las dos anteriores ya que añade la posibilidad de que los usuarios modifiquen los contenidos y añadan semántica básica mediante el uso de etiquetas (tags) que categorizan la información cómo por ejemplo la descripción de un álbum de música mediante el uso de las etiquetas género, autor, entre otras. La Web 3.0 representa un avance muy importante con respecto a sus precursoras ya que nos permite no sólo categorizar la información de forma que pueda ser interpretada automáticamente por los ordenadores sino que además permite relacionar los diferentes contenidos entre sí y crear nueva información mediante el uso de tecnologías como razonadores lógicos (provenientes de la rama de computación de Inteligencia Artificial) o bases de datos de tripletas (Falbo et al). Por ejemplo, si buscáramos artículos sobre Web Semántica escritos por Tim Berners-Lee, con la web tradicional obtendríamos una lista con resultados obtenidos a partir de buscar en la información ocurrencias de las palabras clave 'web', 'semántica' y 'Tim Berners-Lee', los cuales podrían no estar relacionados con la materia en la que estamos interesados, mientras que la tecnología semántica nos permitiría estructurar la búsqueda cómo 'Tim Berners-Lee es el autor del artículo $X$ ' y 'El artículo X menciona la Web Semántica', lo cual produciría resultados más exactos además de permitirnos obtener nueva información cómo 'múestrame artículos de otros autores que han escrito con Tim Berners-Lee sobre Web Semántica'.

El consorcio W3C (organismo internacional para la estandarización de las tecnologías Web) lleva mucho tiempo haciendo hincapié en el gran paso cualitativo que supone la Web Semántica para desenvolvernos en el Internet actual. El mismo consorcio explica como la Web Semántica "no se trata de una inteligencia artificial mágica que permite a las máquinas entender las palabras de los usuarios, es sólo la habilidad de una máquina para resolver problemas bien definidos, a través de operaciones bien definidas que se llevarán a cabo sobre datos existentes bien definidos" (Herman, 2010). 
Esto es posible gracias al desarrollo de una serie de lenguajes informáticos que posibilitan la catalogación, organización, descripción y búsqueda de la información. Entre otros se utilizan RDF (Resource Description Framework), SPARQL (lenguaje utilizado para consultar bases de datos de tripletas o triplestores) y OWL. Siendo este último uno de los lenguajes fundamentales, porque OWL es lenguaje que permite definir ontologías. Las ontologías son el motor de la Web Semántica ya que suponen un vocabulario específico usado para describir una determinada realidad (Falbo et al., 2004). En una ontología (Figura 1), cada conceptualización está basada en conceptos, objetos y las relaciones que se establecen entre ellos. En definitiva las ontologías pueden utilizarse para mejorar la búsqueda de información en la Web, así como para navegar por ella, de tal modo que las ontologías favorecen la interoperabilidad semántica y por eso se utilizan en herramientas de Web Semántica (Abián, 2005). Para definir ontologías hay que tener en cuenta tanto la categorización de conceptos o clases como las relaciones semánticas. La categorización define clases y las relaciones entre ellas. Los elementos concretos son llamados "instancias" de esas clases. Las relaciones semánticas que se definen pueden considerarse predicados que, junto a un conjunto de reglas pueden especificar las clases de las que parte la relación y las clases con las que se asocia según el significado de la misma.

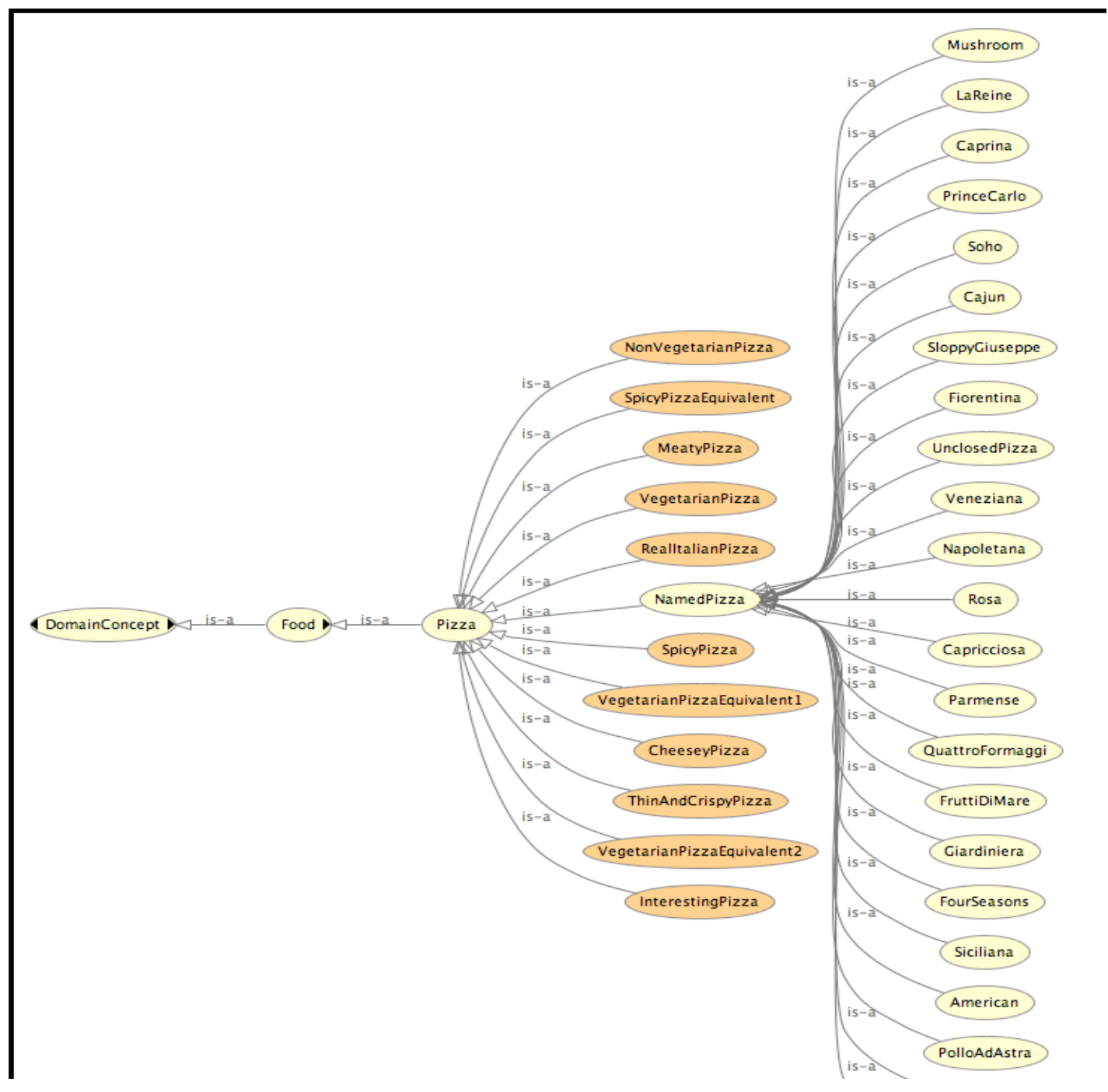

Figura 1. Ejemplo de ontología. Extraído de la ontología "pizza" que contiene el editor de ontologías "Protegé". Visualizado con app OWLViz. 


\section{CÓMO SE TRABAJA CON WEB SEMÁNTICA EN ENTORNOS COMPRENSIVOS: SOFT-ONTOLOGIES}

El proyecto SemTech (Semantic Technologies for Learning and Teaching) trata de identificar los beneficios de las tecnologías semánticas en la educación. En uno de los documentos que ha elaborado este grupo se diferencia entre "hard semantic technologies" (tecnologías semánticas fuertes) y "soft semantic technologies" (tecnologías semánticas blandas) para diferenciar entre las ontologías en función de su nivel de estructuración. Profundizando en este tema, se encuentran autores que hablan del término "lightweight" (Corcho et al., 2003), mientras que otros autores hablan de "soft ontologies" (Aviles et al., 2003) (Tiropanis et al., 2009). De cualquier manera, parece que ambos términos tratan de poder unir diferencias ontológicas aparentemente irreconciliables por medio de análisis contextuales. Esto quiere decir que, cuando se quiere representar el conocimiento de manera estructurada, muchas veces nos encontramos con disciplinas o materias que no pueden ser estructuradas de forma directa en una taxonomía o clasificación. Aquí es donde las ontologías blandas entran en juego.

Siguiendo los informes de Tiropanis et al. (2009) encontramos que:

- Las tecnologías semánticas fuertes se refieren a recursos que son entendidos por las máquinas en su totalidad. Un ejemplo de tecnologías semánticas fuertes sería describir la información utilizando lenguajes como RDF, los cuáles permiten su almacenamiento en bases de datos de tripletas o triplestores (repositorio de metadatos RDF). Si la información se ha estructurado de esta manera, es posible utilizar lo que son conocidos como motores de inferencia y analizar así la información de forma que se genere información nueva aplicando reglas lógicas, mientras que,

- Las tecnologías semánticas blandas o ligeras comprenden la utilización de recursos con la intención de ser mejor entendidos por las personas que por las máquinas. Ejemplo de una anotación semántica blanda sería por ejemplo las folksonomías, muy populares en la Web 2.0 o Web Social. Una de las aplicaciones desarrolladas en el proyecto Ensemble, combina la utilización de ontologías blandas y duras, aplicadas a la disciplina de Danza Contemporánea. Esta aplicación permite tanto a alumnos como profesores anotar vídeos e imágenes de sus actuaciones utilizando términos similares a los utilizados en las folksonomías (ontologías blandas) combinados con términos provenientes de ontologías formales en el ámbito de la danza como el vocabulario de Laban.

Muchas de las tecnologías 2.0 para educación utilizan tecnologías semánticas blandas como wikis y tags (etiquetas), entre otras, aunque al mismo tiempo, hay que encontrar un punto intermedio entre las tecnologías semánticas blandas y las duras para poder desarrollar aplicaciones realmente útiles. Un ejemplo de tecnología semántica blanda es la Wikipedia y un ejemplo de tecnología semántica dura basada en una estructura (RDF) es DBPedia o FreeBase (Tiropanis et al., 2009).

Algo parecido ocurre con el concepto de ontología, hemos definido previamente la ontología como "una teoría lógica constituida a partir de un vocabulario y un lenguaje lógico" 
(Maetdche, 2002). En función de cómo elaboremos nuestra ontología podemos hablar del mismo modo de ontologías más o menos ligeras:

- Ontologías fuertes: ontologías fuertes son las que están estructuradas a través de lenguajes específicos como pueden ser RDF o OWL. Normalmente se desarrollan a través de editores de ontologías como Protegé.

- Ontologías ligeras: una ontología ligera se refiere a una ontología flexible, se refiere a organizaciones conceptuales que conllevan a un dominio de términos flexibles, sirve para presentar un contenido sin tener que desarrollar una taxonomía o una clasificación exhaustiva (Avilés et al, 2003). Un ejemplo de ontologías blandas sería la definición de conceptos utilizando el lenguaje SKOS (Miles \& Bechhofer, 2009).

Es destacable la propuesta que hacen Corcho et al. (2003) al mencionar, por un lado, las ontologías "lightweight", que incluyen los conceptos, las taxonomías de los conceptos, las relaciones entre conceptos y las propiedades que describen los conceptos. Por otra parte, se encuentran las ontologías "heavyweight" y que sí superan el ámbito de los tesauros contemplados anteriormente en la medida que añaden axiomas.

Hay otro concepto relacionado, que es el de "weak ontology". Una ontología débil es la que no es lo suficientemente rigurosa para permitir que el software la interprete.

Que una ontología sea ligera no significa que sea menos eficaz. Realmente hay áreas en las que es difícil establecer unas relaciones claras. El desarrollo de aplicaciones que permiten crear lo que los autores denominan "soft-ontologies" o "lightweight ontologies" también ha aparecido en contraposición al complicado desarrollo que requieren las ontologías. Una de las aplicaciones más importantes desarrolladas en este sentido es Exhibit. Exhibit es una aplicación del proyecto SIMILE (Semantic Interoperability of Metadata and Information in unLike Environments). Es proyecto investiga en el campo de la interoperabilidad semántica de metadatos, i.e. derrallodo de aplicaciones de gestión de la información en diferentes entornos (MIT, ). SIMILE pretende mejorar la interoperabilidad entre los activos digitales, los esquemas, el vocabulario, las ontologías, los metadatos y los distintos servicios. El software que desarrollan es de código abierto, es decir, es software libre (bajo una licencia BSD).

Huynh et al. (2008) afirman que la Web Semántica ha sido desestimada por muchos basándose en que tenía una visión poco realista de la realidad, ya que se lanzó como un medio en el que los agentes de software automatizado de recolección de datos estructurados llevaban a cabo tareas complejas de forma automatizada. Estos autores intentan demostrar con Exhibit que, incluso sin agentes automatizados, hay un enorme valor en la normalización de un modelo de datos estructurado entre los objetos con propiedades y valores. Dicha normalización ofrece una forma limpia y reutilizable de separar el contenido de la presentación, y permite la presentación de contenido en formas más interactivas a partir de una gran variedad de información .

Algunos seguidores de la Web Semántica con una visión más tradicional o estricta podrían preguntar dónde están las ontologías OWL y cómo se incluirían en estos modelos abiertos. Huynh et al. (2008) nos dan la respuesta indicando que han escogido un modelo de sintaxis diferente, que no se basa en RDF/XML, ellos aceptan las limitaciones de no trabajar con estas tecnologías y lo asumen, porque por el contrario el método de trabajo de Exhibit les permite desarrollar aplicaciones de forma más rápida, práctica y accesible a un mayor 
número de usuarios. En cuanto al OWL, argumentan que de momento es contraproducente intentar apuntar tan alto hacia formas plenamente automatizadas. Desde su perspectiva, el primer paso crítico para la generalización de la Web Semántica es la de generar grandes cantidades de información estructurada en la forma adecuada y Exhibit es una herramienta que establece las bases para lograr este objetivo. La racionalización de los datos puede venir después:

"La Web temprana (Web 1.0) era anárquica, sólo después fue domesticada por los directorios y motores de búsqueda. Del mismo modo, la Web Semántica temprana es probable que sea una entidad caótica estructurada cuya existencia puede motivar el desarrollo de sofisticadas herramientas de alineamiento y razonamiento que puedan traer el orden a ella" (Huynh, Karger y Miller, 2008:9).

A continuación se muestran algunos ejemplos de recursos electrónicos creados con la herramienta Exhibit (Figuras 2, 3 y 4):

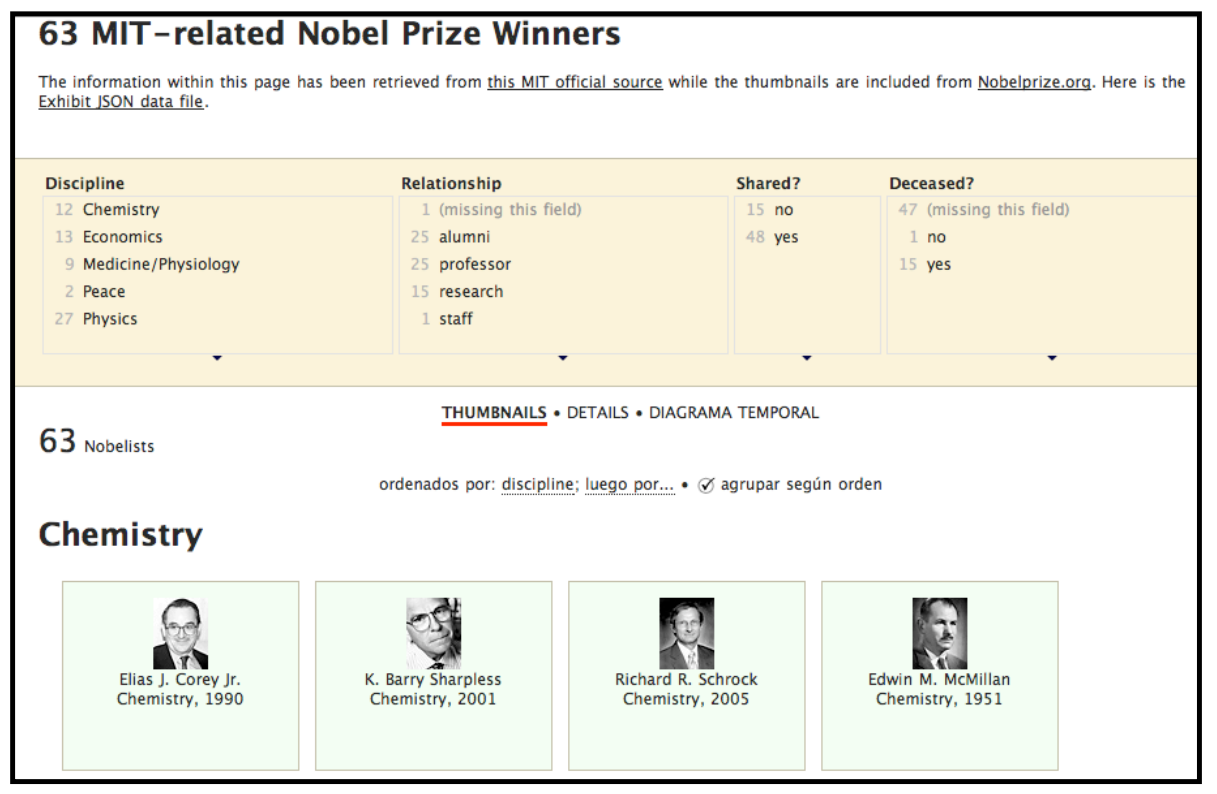

Figura 2: ejemplo de página web que utiliza la herramienta Exhibit desarrollada por el MIT. Ganadores del MIT del premio Nobel. (http://simile-widgets.org/exhibit/examples/nobelists/nobelists.html) 


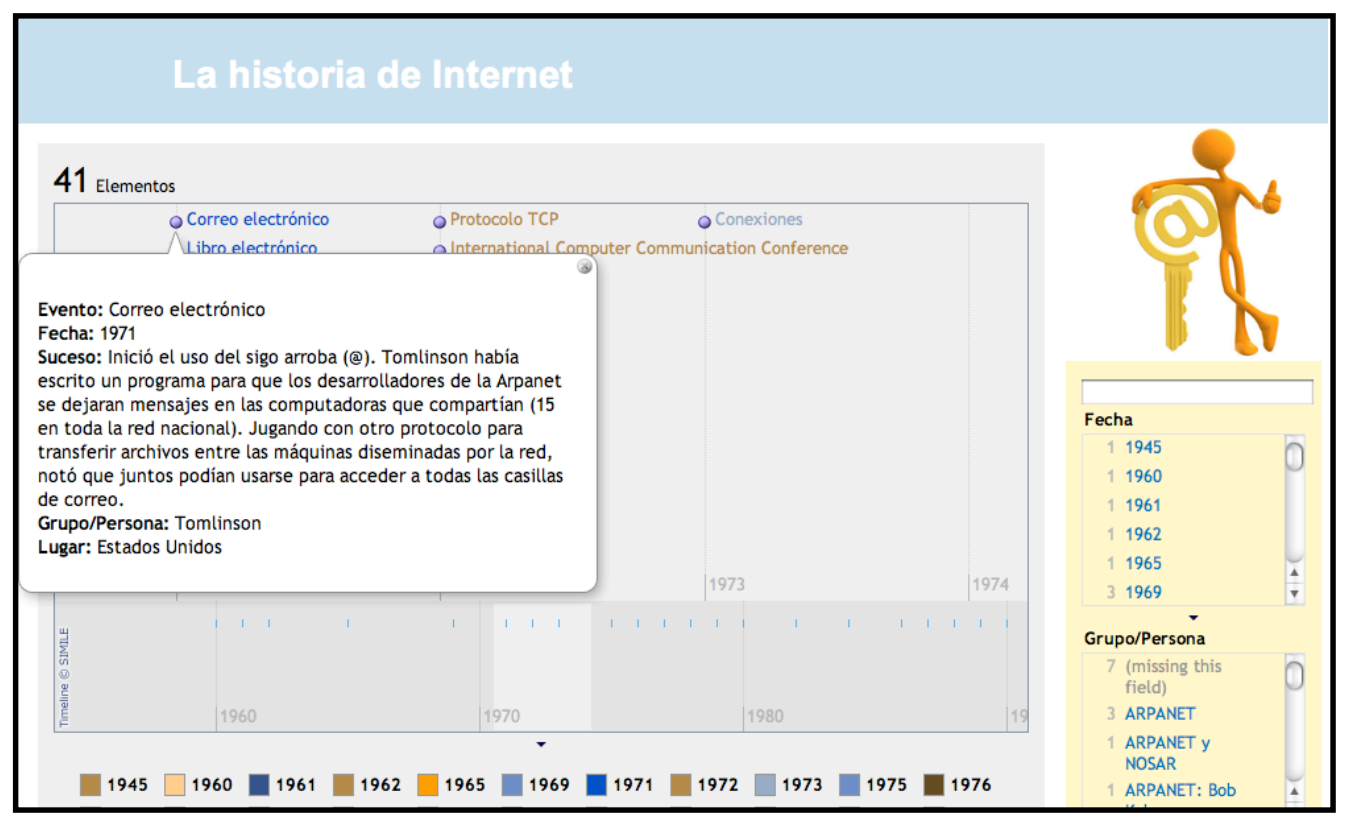

Figura 3: ejemplo de página web propia creada con Exhibit con línea del tiempo incluida. La historia de Internet.

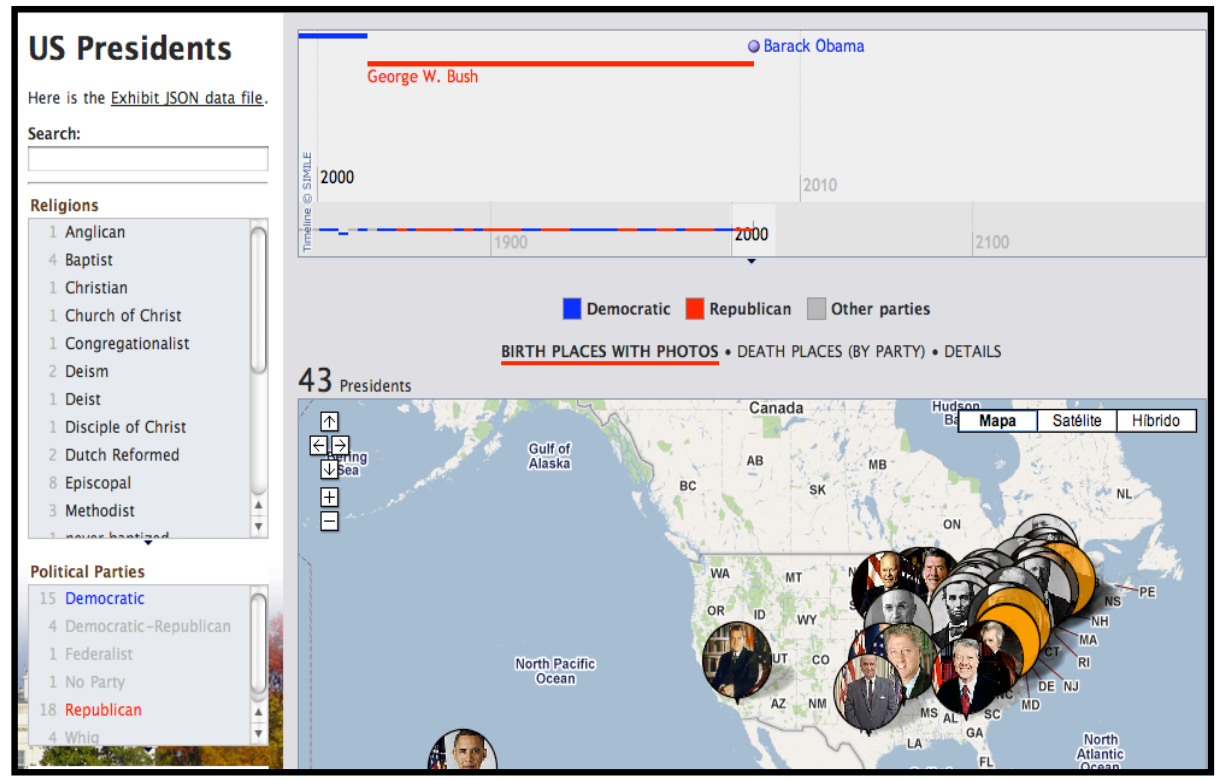

Figura 4: ejemplo de página web que utiliza Exhibit y Google Maps. Presidentes de EEUU. (http://similewidgets.org/exhibit/examples/presidents/presidents.html)

\section{EXPERIENCIAS DE WEB SEMÁNTICA EN LA EDUCACIÓN}

La Web Semántica es una temática de actualidad, ésa es la conclusión principal a la que podríamos llegar al contemplar la gran cantidad de información e investigaciones encontramos respecto al tema. En este sentido, Castells (2003) confirma que la Web Semántica se ha convertido en un área de investigación de moda, ya que es un tema de interés de centros de investigación de todo el mundo, entre ellos, el MIT, la Universidad de Standford, la Universidad de Maryland, la Universidad de Innsbruck, la Universidad de Karlsruhe, la Universidad de Manchester y la Open University del Reino Unido. 
Breis et al. (2007) se plantean que la importancia de las propuestas de la Web Semántica ha sido reconocida por las distintas entidades nacionales y supranacionales en materia de desarrollo tecnológico e indican la importancia de las mismas a distintos niveles (Breis et al., 2007):

- Tanto el gobierno de Estados Unidos (DARPA) como la Unión Europea (Programa Marco) están financiando investigaciones encaminadas al desarrollo de la tecnología base para la Web Semántica. Tanto en el VI como en el VII Programa Marcos las áreas semánticas han recibido más de 50 mil millones de euros. Existen empresas y centros de investigación en todo el mundo dedicando una importante cantidad de recursos a la Web Semántica. Las grandes multinacionales como IBM, Microsoft, Sun, Oracle, O HP están desarrollando activamente estándares y tecnologías para la Web Semántica. Centros como MIT o la Universidad de Stanford en Estados Unidos, la Universidad de Karlsruhe (Alemania) o el Digital Enterprise Research Institute (sedes en Austria e Irlanda) son algunos de los más importantes.

- A nivel nacional y regional también existe cada vez mayor concienciación de la importancia de las tecnologías de la Web semántica. Las tecnologías de la Web Semántica aparecen por primera vez en el Plan Regional de Ciencia y Tecnología 2007-2010. En el año 2007 se ha puesto en marcha la primera Red Temática Nacional sobre Web Semántica, coordinada por Asunción Gómez Pérez de la Universidad Politécnica de Madrid. Esta red está compuesta por 27 grupos de investigación nacionales que cubren prácticamente toda la geografía peninsular. Entre las universidades representadas podemos mencionar la Universidad de Murcia, la Universidad Autónoma de Madrid, la Universidad Carlos III, la Universidad de Oviedo, la Universidad de Extremadura, la Universidad de Santiago de Compostela, la Universidad Rovira i Virgili o la Universidad de Deusto.

- En el ámbito empresarial también existe interés en nuestro territorio nacional. La empresa española ISOCO es posiblemente la empresa europea más importante en el ámbito de la Web Semántica, habiendo realizado proyectos que emplean estas tecnologías en diversos ámbitos como trámites electrónicos, banca o patrimonio cultural. Sin embargo, no es la única empresa que ha empleado con éxito estas tecnologías en nuestro territorio, ya que Ximetrix es otro ejemplo de la viabilidad industrial de estas tecnologías.

Muchas Universidades y centros de investigación institucionales se encuentran inmersos en la investigación de la Web Semántica, y muchos de ellos concretamente en el ámbito educativo.

En primer lugar, es destacable el trabajo desarrollado por el grupo TECNOMOD y el GITE. EI grupo TECNOMOD (Grupo de Tecnologías de Modelado, procesamiento y gestión del conocimiento $)^{1}$ de la Universidad de Murcia liderado por Jesualdo Tomás Fernández Breis ha desarrollado la aplicación OeLE (Ontology elearning Environment) basada en Web Semántica para la creación de entornos de evaluación basados en preguntas abiertas gracias al cual es posible desarrollar este trabajo. EI GITE (Grupo de Investigación de Tecnología Educativa) $)^{2}$, dirigido por $\mathrm{M}^{a}$ Paz Prendes Espinosa, trabaja estrechamente con el grupo

\footnotetext{
${ }^{1}$ TECNOMOD: https://curie.um.es/curie/catalogo-grupo-investigacion.du?cods=E096*03

2 GITE: http://www.um.es/gite
} 
TECNOMOD, liderado por Jesualdo Tomás Fernández Breis, para valorar las implicaciones educativas de la aplicación de OeLE. Este proyecto se centró en el proceso de evaluación de las intervenciones y exámenes que se brinda en los entornos educativos, de forma que se mejoren los procesos colaborativos entre los estudiantes, y entre éstos y los profesores. De esta forma, el profesor tendría que dedicar menos tiempo a la evaluación de las preguntas abiertas, apoyándose en una conceptualización del dominio del conocimiento y en técnicas de la Web Semántica. La principal novedad de este proyecto radica en hacer confluir varias técnicas y tecnologías actuales de la Web Semántica y el Procesamiento del Lenguaje Natural con el propósito de semi-automatizar el proceso de evaluación en eLearning. El trabajo desarrollado ha sido posible gracias al Proyecto 08756/PI/08 "Plataforma Semántica de Formación a la carta" financiado por la Fundación Séneca de la Región de Murcia y a la beca de Formación de Profesorado Universitario (FPU) del Ministerio de Educación de España. Su finalidad principal es hacer uso de de las tecnologías Java y de la Web Semántica para integrar el módulo de trabajo en grupo en la plataforma existente (OeLE) para que un profesor pueda combinar pruebas individuales y colectivas en procesos de evaluación continua.

Además de este trabajo, a nivel de Educación Superior es interesante detenerse en un proyecto de investigación relevante debido que es uno de los proyectos más importantes en lo referido a financiación y equipo investigador que trabaja con Web Semántica y Educación. Hablamos del proyecto ENSEMBLE, "Tecnologías Semánticas para la mejora del aprendizaje basado en problemas". ENSEMBLE es un proyecto internacional financiado conjuntamente por el Consejo Económico y Social y el Consejo de Investigación de Ingeniería y Ciencias Físicas del Consejo de Investigación en el marco del Programa para le mejora del aprendizaje en TIC. Este proyecto está financiado con 1 millón de libras esterlinas; dirigido por el Dr. Patrick Carmichael reúne a investigadores educativos y tecnólogos de la Universidad Liverpool John Moores, la Universidad de Cambridge, la City University de Londres, la Universidad de Stirling, la Universidad de East Anglia y la Universidad de Essex, junto con colaboradores del Instituto Tecnológico de Massachusets (MIT) y la Universidad UTS de Sydney. El proyecto ENSEMBLE es uno de los proyectos internacionales más importantes que estudia la aplicación de la Web Semántica en la Educación. El proyecto ENSEMBLE está investigando la aplicación de tecnologías semánticas en entornos de aprendizaje basado en casos. Lo que principalmente le diferencia de otras investigaciones de educación y Web semántica es que aborda la investigación de la Web Semántica desde una perspectiva educativa y trata de ver qué implicaciones conlleva su uso en la educación, mientras que otros grupos de investigación se centran en aspectos técnicos o informáticos de la Web Semántica. El proyecto trabaja con los profesores y estudiantes de pregrado y posgrado de las universidades mencionadas para explorar en qué aspectos se centra el aprendizaje, y qué papel pueden desempeñar las nuevas tecnologías de Web Semántica en el apoyo a este aprendizaje. En este momento se han creado ya aplicaciones educativas que se pueden observar en la página Web del proyecto (http://www.ensemble.ac.uk/).

Tscholl et al. (2009) proporcionan una revisión de las características de las diferentes asignaturas y programas en los que se está investigando y llevando a cabo, por un lado un análisis de los aspectos pedagógicos del aprendizaje basado en casos y por otro las diferentes aplicaciones tecnológicas que se están desarrollando hasta el momento en el proyecto. 
Los investigadores de Ensemble han estado trabajando con profesores e investigadores educativos con el fin de reflexionar y apoyar sus prácticas pedagógicas. Recientemente, el coordinador educativo y los profesores en el departamento pusieron de manifiesto la necesidad de un cambio en la estructura de uno de los módulos del curso, concretamente un módulo sobre Biocombustibles, el cual cuenta con la participación de investigadores y consultores expertos en el área y está enmarcado en el tercer año del grado. La motivación fundamental para este cambio ha sido por un lado permitir la integración de un enfoque centrado en el alumno como una alternativa a las clases magistrales y por otro, favorecer las actividades colaborativas a la par que autónomas de los alumnos ya que el objetivo es escribir in informe de investigación sobre un tema dado y realizar una presentación final. De este modo se espera que los estudiantes adquieran conocimientos expertos sobre problemas del mundo real, fomentando así sus capacidades profesionales, destacando entre otras: habilidad para el trabajo colaborativo, el análisis de datos auténticos, la colaboración con otros expertos, la reflexión sobre las prácticas, y otras competencias sobre el uso de las TIC, como hacer presentaciones y la redacción de informes. El módulo, centrado en la investigación de los nuevos biocombustibles basados en las algas, trata temas muy actuales en este área ya que evoluciona rápidamente. Debido a esto último, uno de los objectivos principales del módulo es que los alumnos adquieran experiencia en la evaluación crítica de artículos de investigación actuales y hagan uso de ellos en los trabajos escritos y las presentaciones que tienen que realizar al final del módulo. Por otro lado, una cuestión fundamental para los profesores del módulo es que los alumnos se centren en los aspectos científicos, relevando así a un segundo plano otras cuestiones secundarias como las implicaciones políticas y económicas del uso de biocombustibles.

Con respecto al uso de tecnologías, y como apoyo en sus actividades de aprendizaje, los alumnos utilizan un entorno de aprendizaje en red (Virtual Learning Environment - VLE), el cual dispone de una serie de herramientas de colaboración (no semánticas). EI VLE proporciona tanto un espacio de almacenamiento de archivos como una serie de herramientas comunicativas y colaborativas. Por otro lado, un entorno wiki fue creado para grupos de estudiantes como sistema de publicación de sus informes y una herramienta de blogs permitía a los estudiantes reflexionar sobre su investigación y compartir ideas fuera de las horas de reunión del grupo. Las observaciones de los alumnos durante sus actividades de aprendizaje, entrevistas con los profesores del módulo y un focus Group final con los estudiantes indicaron dos áreas en las cuales Ensemble podría implicarse y aplicar tecnologías semánticas como soporte a las actividades del módulo: herramientas de colaboración en línea y herramientas para la gestión de referencias bibliográficas además de permitir recomendaciones. Con respecto al primer grupo, el feedback obtenido reveló que los estudiantes necesitaban más apoyo en línea para compartir recursos y poder fomentar así la continuación de los debates fuera de las reuniones del grupo y con respecto a la segunda, los alumnos necesitaban apoyo no sólo a la hora de encontrar referencias bibliográficas útiles sino también de cara a compartir dichas referencias. Se consideró que la Web Semántica podía ayudar a trabajar con la información y mejorar las aplicaciones para la gestión de los recursos. Las conversaciones con los organizadores del curso destacaron la necesidad de los estudiantes de conservar los vínculos con la información y las conclusiones que hicieron en la presentación del caso y en el informe inicial, así como los recursos que han trabajado. De este modo, la presentación final de los casos se ha realizado utilizando una herramienta de visualización semántica (como los que demuestra SIMILE en el MIT 
vistos anteriormente) la cual es respaldada con otras herramientas que permiten vínculos más claros a los datos originales y las fuentes académicas.

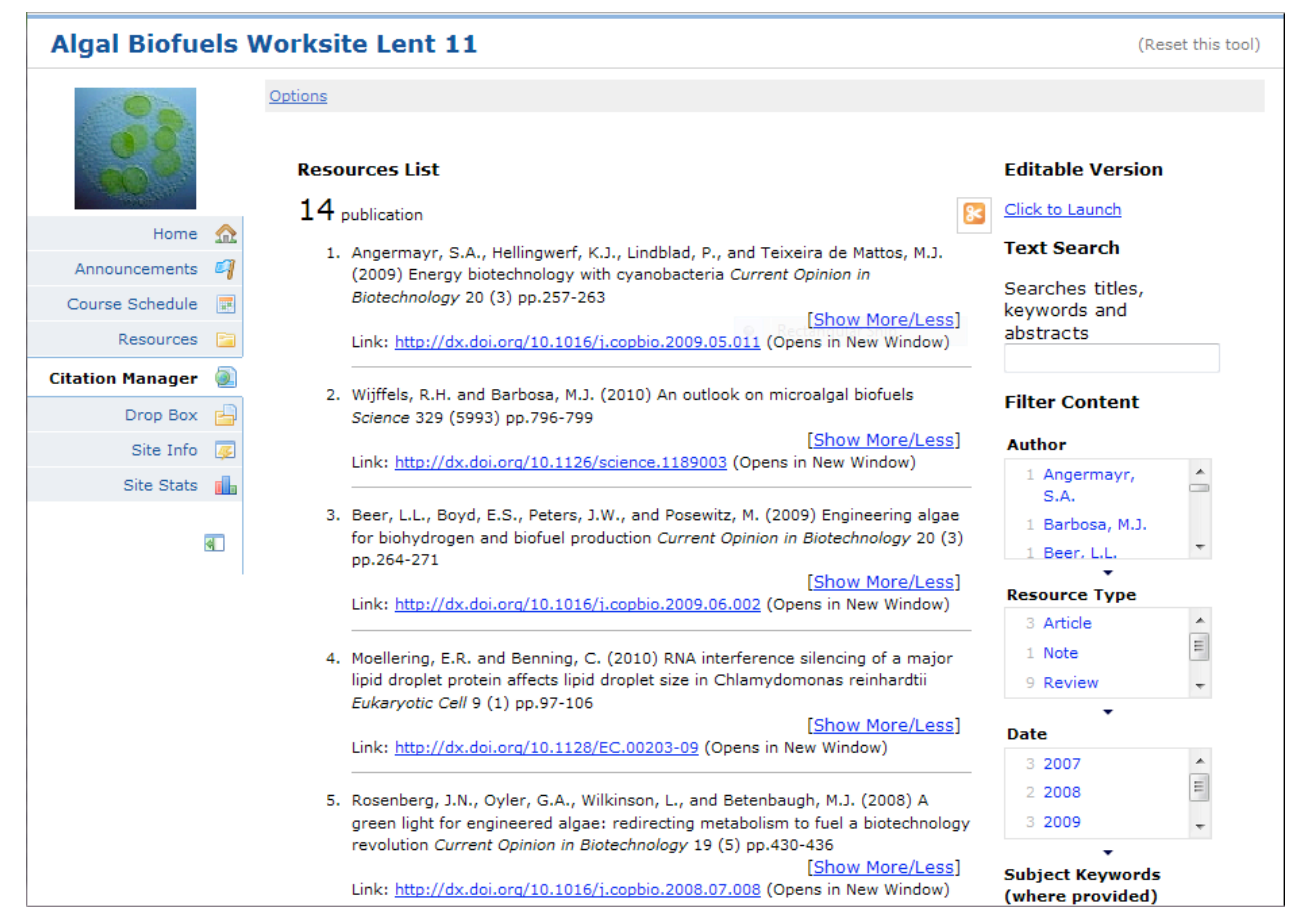

Figura 5: Navegador temático, integrado en el EVA, junto con otras herramientas de soporte al alumno

\section{Arqueología y antropología}

Esta materia se imparte gracias a la colaboración de una amplia gama de instituciones y centros, entre ellos, además de la propia Facultad de Arqueología y Antropología encontramos, por ejemplo, el Museo de Arqueología. El grupo de ENSEMBLE se reunió en numerosas ocasiones con el grupo de profesores involucrados en el grado de Arqueología. En esta asignatura los profesores se apoyan en un conjunto de casos que son utilizados para facilitar las tareas de clasificación de materiales y objetos encontrados en las excavaciones. Por otro lado, los alumnos 'contruyen' casos, los cuales reflejan el proceso que han llevado a cabo cuando examinaban, registraban y publicaban los materiales excavados. El equipo de ENSEMBLE se ha encontrado con una gran variedad de opiniones y perspectivas sobre la motivación que hay detrás de las prácticas pedagógicas de enseñanza (más concretamente el uso de casos) aplicadas en la asignatura. Por un lado, los profesores construyen los casos en base a su experiencia cuando formaban parte de equipos profesionales de arqueólogos realizando expediciones mientras que por el otro, los casos reflejan los procedimientos empleados en Museos, centros de restauración relacionados con la conservación de materiales arqueológicos.

Después de analizar y realizar numerosas observaciones tanto de alumnos como de profesores durante las actividades educativas y después de dialogar con los profesores implicados, se decidió introducir elementos tecnológicos en un módulo del último año. La actividad a realizar por los alumnos consiste en el desarrollo de un proyecto final sobre un artefacto del museo. Los alumnos deben realizar un estudio sobre un artefacto del museo y presentar un informe final. Esta actividad presenta similitudes con las actividades reflejadas 
en el ejemplo anterior ya que los alumnos trabajan con fuentes de información diversa, la cual se integra en sus informes finales.

Los proyectos de los alumnos resultaron muy apropiados de cara a la introducción de tecnologías semánticas ya que permiten trabajan con información y añaden interactividad a la presentación. La aplicación desarrollada consiste en un una aplicación online que permite visualizar de forma integrada fragmentos del análisis llevado a cabo por el alumno junto con información obtenida en tiempo real del archivo del museo o elementos visuales como fotografías, mapas, ...; obtenidos a través sitios especializados en Arqueología disponibles online. El mejor ejemplo para ilustrar las mejoras introducidas por este tipo de tecnologías se basa en un proyecto en concreto de uno de los alumnos. El informe realizado por el alumno recopila una pequeña colección de broches anglosajones encontrados durante una excavación llevada a cabo en el Reino Unido. El informe del alumno se reconstruyó de forma que permitiese integrar las reflexiones y análisis del alumno con contenidos extraídos de la base de datos del museo de Arqueología y otras fuentes externas. Esto permitió enriquecer sus reflexiones con elementos multimedia variados destacando:

- Foto galeria que incluye imágenes tanto históricas como contemporáneas del sitio de excavación.

- Mapa interactivo, el cual muestra excavaciones similares realizadas en otras regiones.

- Tabla ilustrativa de la tipología de broches anglosajones.

- Bibliografía con enlaces a materiales complemenarios relacionados

Magisterio (Education Studies)

El hecho de que los estudiantes de Magisterio trabajasen con fuentes de información diversa y las relacionasen con sus propias experiencias fue la motivación principal para trabajar de forma conjunta con alumnos y profesores. En uno de los módulos de la asignatura de 'Historia de la Filosofía', la cual se enmarca en el primer curso del grado, se requiere que los alumnos sean capaces de relacionar políticas actuales con prácticas en el ámbito educativo a la par que situar ambas en los marcos histórico-filosóficos de la educación. Los estudiantes encontraron esta tarea extremadamente complicada y mientras que por un lado, el aprendizaje basado en casos (tanto proporcionados por los profesores como elaborados por los alumnos) representaba parte de la solución al problema por el otro, el papel de las tecnologías semánticas estaba todavía por explorar.

Tras realizar varias actividades de diseño en las que participaron investigadores del grupo y profesores de la asignatura, se decidió que la aplicación más apropiada de cara a organizar la información del módulo era una 'línea del tiempo interactiva' (Figura 6). Esta aplicación permite situar recursos de aprendizaje no solo en relación a objetivos de aprendizaje y actividades sino que también permite situarlos en un marco histórico más amplio. Algunos ejemplos de contenidos incluidos en la línea del tiempo son: gobiernos, personalidades políticas y los desarrollos sociales llevados a cabo además de informes sobre legislación educativa. 


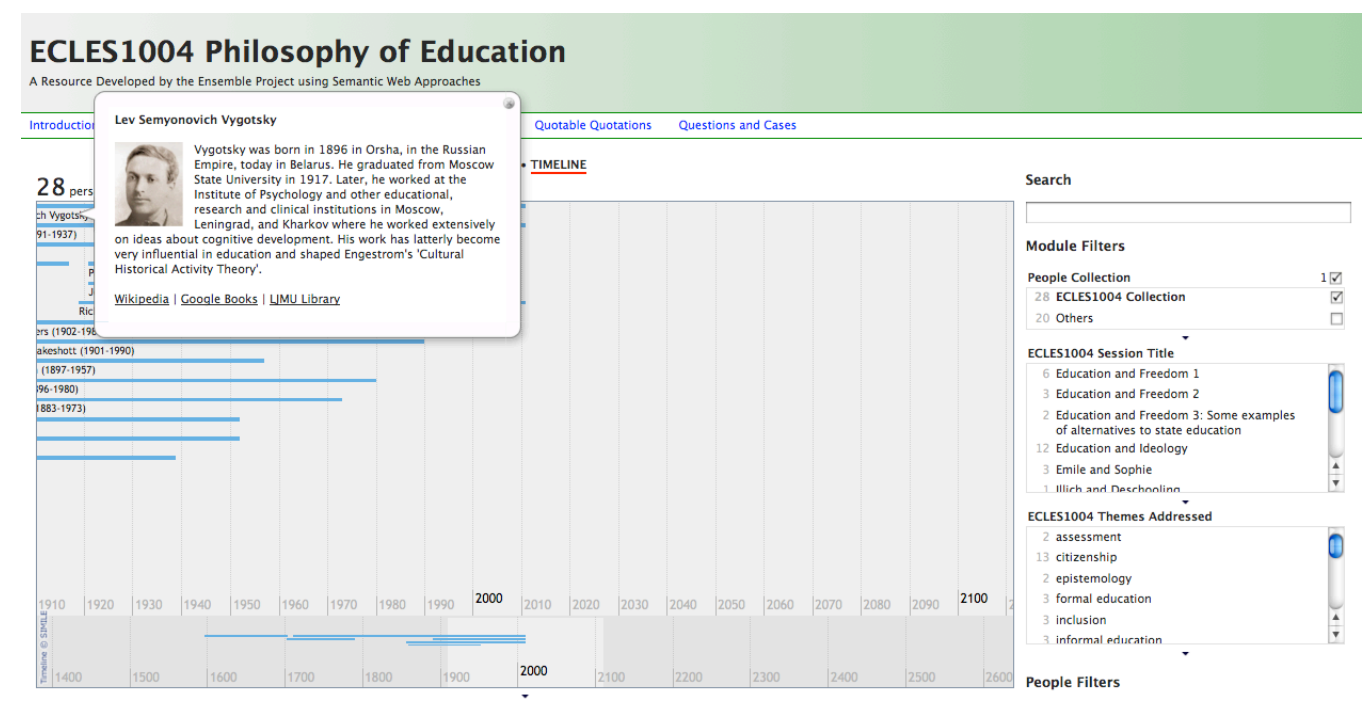

Figura 6. Línea del Tiempo Interactiva como mecanismo para organizar los contenidos con temas educativos y casos.

Operaciones Marítimas y gestión (MOAM)

MOAM (Maritime Operations and Management) es una asignatura sobre operaciones marítimas y gestión. El contenido trata sobre la industria marítima. El procedimiento de trabajo realizado desde ENSEMBLE ha sido el de realizar varias entrevistas a profesores y alumnos para conocer cómo funciona el curso y un estudio de los materiales que se utilizan en la asignatura a distintos niveles. En esta materia los alumnos trabajan en las prácticas con diferentes casos reales, que formulan un problema al cual ellos tienen que saber darle una solución práctica. Son escenarios de aprendizaje, por ejemplo, una tarea puede ser diseñar un servicio de Ferry desde un archipiélago hasta la costa de Cornualles. Los alumnos tienen que jugar el papel de expertos en distintos dominios (economistas, ingenieros de seguridad, diseñadores técnicos) y presentar y defender su diseño de trabajo ante un panel de expertos. Esta forma de utilizar los casos requiere un enfoque para pensar en el apoyo tecnológico de herramientas semánticas.

Un primer acercamiento ha sido tratar de realizar una ontología sobre como funciona el curso. Cuando antes se hablaba de un estudio de los materiales a distintos niveles se hace referencia a que se han contemplado también los distintos objetivos que se proponen realizar en el curso. Las programaciones aparecen a distintos niveles. Tenemos por un lado, los objetivos que tiene la universidad para esa asignatura a nivel institucional, los objetivos del departamento y los objetivos que formula el profesor en su material y en cada tema. Un mapa conceptual intenta en primer lugar organizar toda esta información: 


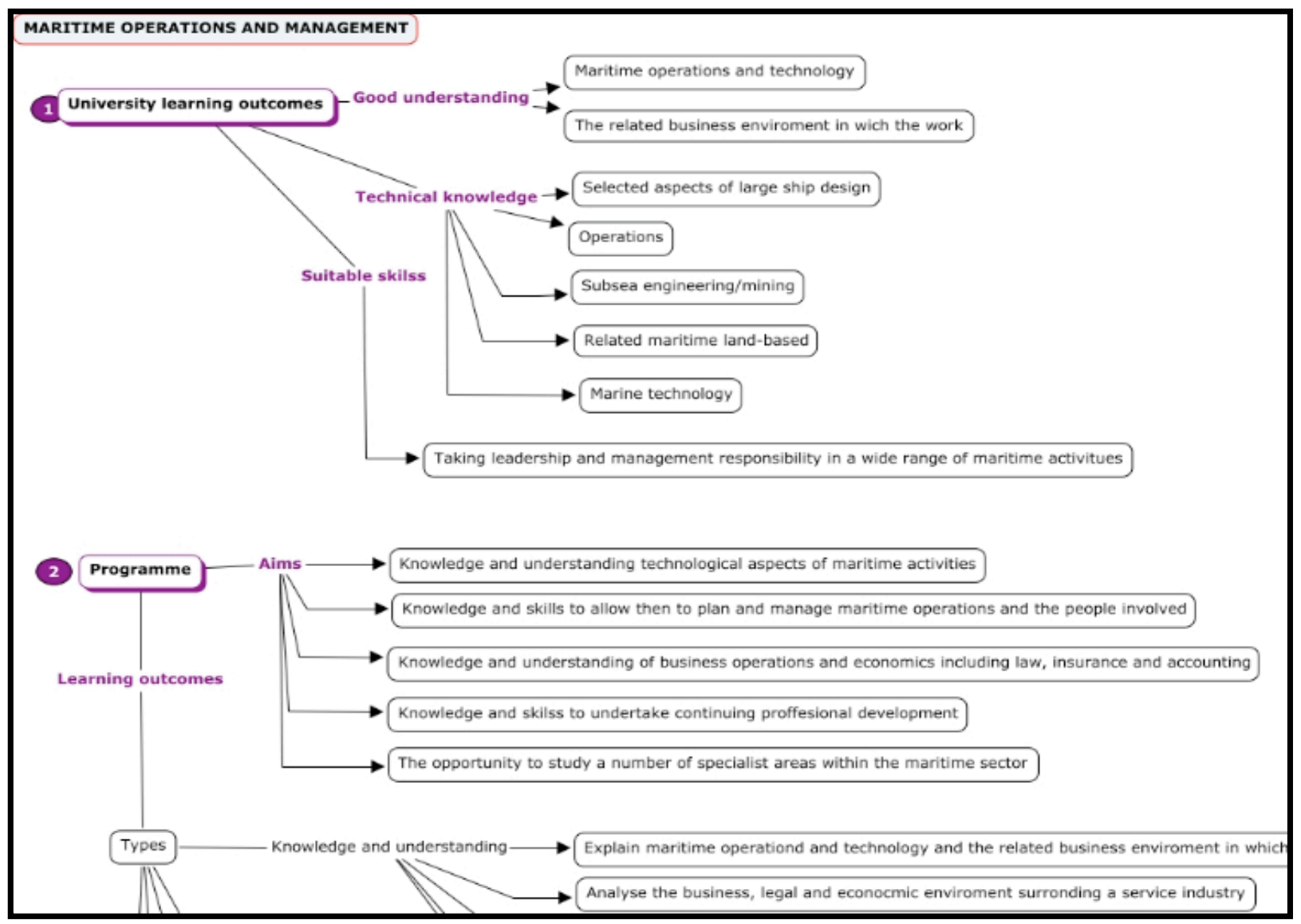

Figura 7: ejemplo de mapa elaborado con Cmaps para organizar el curso MOAM

Por otro lado, recoger los objetivos de una asignatura en una ontología no es una tarea fácil, y más si queremos que nuestra ontología sea estable en el tiempo y pueda servir para otros objetivos, por tanto, a través de una taxonomía educativa se trató de diferenciar los distintos elementos que confluyen en los objetivos (acción, contenido, etc.) y relacionarlo estructuralmente con hojas de cálculo en los que la información se encuentra estructurada y pueda ser relacionada para crear aplicaciones semánticas que traten de conocer cómo funciona un curso, y no únicamente restringirnos a aspectos de contenido. Esto puede ofrecernos diversas aplicaciones, entre ellas por ejemplo, una en la que un profesor pueda buscar por palabras clave objetivos y conocer cómo fueron desarrollados, por medio de qué tareas y al mismo tiempo movernos por una ontología del curso que nos permita conocer, por ejemplo, si este objetivo responde a un enfoque institucional o depende de la planificación del profesor.

Otras investigaciones que se están realizando sobre Web Semántica y educación son las siguientes:

- Universidad Autónoma de Madrid, Grupo de Herramientas Interactivas Avanzadas (GHIA, ). Este grupo de investigación está desarrollando la aplicación ATENEA. Esta aplicación se basa en el uso de técnicas estadísticas y de procesamiento del lenguaje natural para procesar las respuestas de texto libre aunque no hace uso de tecnologías semánticas. 
- Free University Amsterdam (FUA, 2011). Están trabajando en la aplicación de Tecnologías de la Web Semántica en el eLearning y e-cultura mediante estructuras conceptuales en educación e investigación en sistemas de recomendación.

- Stanford Center for Innovations in Learning (SCIL, ): investigan en diversos tópicos de eLearning entre los que incluyen la Web Semántica como aspecto fundamental de la Web y la educación.

- Liverpool John Moores University: Patrick Carmichael que trabaja, entre otras instituciones, con CARET (Centro de Nuevas Tecnologías de la Universidad de Cambridge) y la City University de Londres, lidera el proyecto ENSEMBLE (Ensemble, ), el cual está investigando y desarrollando aplicaciones semánticas para el aprendizaje basado en casos.

- Universidad de Karlsruhe (University, 2010) investiga en la aplicación de las tecnologías del conocimiento y la Web Semántica en diferentes campos siendo el elearning uno de ellos.

- Universidad de Hannover, el Learning Lab, Institut fur Informationssysteme (LLII, ) está investigando en el campo del e-learning, Web Semántica y bibliotecas digitales, entre otros proyectos.

- Universidad de Winston-Salem State, la Universidad de Pittsburgh y la Universidad Saint-Petersburg State Polytechnic participan en el proyecto SWEL (Ontologies: Social Semantic Web for Education) (o4e, ). Están desarrollando el portal O4E, cuyo objetivo principal es recopilar y estructurar la información disponible relacionada con el uso de ontologías en el campo de la educación. Destacar que el propio portal está dirigido por una ontología desarrollada en el proyecto y que permite navegar por todos los contenidos relacionados con ontologías y web semántica recopilados hasta el momento.

En el sector privado encontramos también diversas inversiones, como la del proyecto Halo (http://www.projecthalo.com) financiado por la empresa Vulcan INc (propiedad de Paul Allen). El proyecto Halo tiene como finalidad el desarrollo de un sistema basado en tecnologías de la Web Semántica y del conocimiento capaz de responder a cualquier cuestión que se le plantee. Se persigue que sea un tutor capaz de instruir y evaluar a los estudiantes de ciencias y que sea un asistente científico con habilidades inter-disciplinares. Este proyecto se basa en la capacitación profesional. En 2007 se amplió con el proyecto Halo de Investigación Avanzada (Halar), para abordar la difícil representación del conocimiento y el razonamiento. En la página Web se puede encontrar un lenguaje diseñado a partir de reglas semánticas y un sistema de razonamiento de inferencia semántica en el conocimiento de gran tamaño.

\section{CONCLUSIONES Y DISCUSIÓN}

Podemos identificar dos temáticas en las investigaciones; las que se centran en la Web semántica y la educación que intentan aplicar herramientas semánticas a entornos educativos y conocer su funcionamiento; y además específicamente podemos indicar una serie de investigaciones sobre la evaluación en entornos virtuales, incluyendo en este punto los sistemas de evaluación en red tradicionales y los sistemas de evaluación basados en la 
semántica y otras técnicas. De cualquier modo es destacable la importancia de abordar el tema de la Web Semántica desde la educación.

La web es utilizada en educación, ya en 1995 afirmaban Ibrahim y Franklin sus potencialidades debido a que presenta características como:

- El hipertexto que permite estructurar la información de forma no lineal, sino multidimensional, haciendo que los participantes construyan el significado en la dirección que consideran más atractiva e interesante.

- La capacidad multimedia, ya que en la Web se permite el intercambio de documentos en diferentes formatos estándares.

- La ubicuidad que otorga el ser un sistema distribuido y abierto a Internet, lo cual facilita el acceso a los documentos contenidos en esta.

- La posibilidad de formar grupos de trabajo colaborativo a través de diversas aplicaciones.

¿Qué características podría aportar entonces la Web Semántica a la educación?

- La organización semántica de la información proporciona la posibilidad de trabajar con aplicaciones organizadas de un modo más comprensivo, lo que facilitaría la utilización de la misma en cualquier proceso educativo favoreciendo la organización de la información.

- La aplicación semántica a sistemas de evaluación en red proporcionaría la posibilidad de realizar exámenes de preguntas abiertas que fueran corregidas por las aplicaciones semánticas a partir de las ontologías.

- La inclusión de la Web Semántica en motores de búsqueda y organización posibilitaría búsquedas más personalizadas y coherentes. La unión de esta posibilidad junto con la de los Objetos de Aprendizaje posibilitaría la creación de sistemas de aprendizaje "a la carta", personalizados en función de las necesidades del usuario o de sus resultados previos.

\section{BIBLIOGRAFÍA}

ABIÁN, M. A. (2005). El futuro de la Web: XML, RDF/RDFS, ontologías y la Web Semántica. XML.com. Documento electrónico, http://www.hosteltur.com/fdb/El_futuro_de_la_Web.pdf [Consultado el 19 de Noviembre de 2010]

ALLERT, H., MARKKANEN, H., \& RICHTER, C. (2006). Rethinking the Use of Ontologies. En Proceedings of the 2 nd International Workshop on Learner-Oriented Knowledge Management and KM-Oriented Learning (LOKMOL 06) (pp. 115--125).

AVILES, K., DIZA-KOMMONEN, L., LAIPAINEN, M., \& PIETARILA, J. (2003). Soft Ontologies and Similarity Cluster Tools to facilitate Exploration and Discovery of Cultural Heritage Resources. IEEE Computer Society Digital Library. 
BREIS, J. F., ESPINOSA, M. P. P., NIEVES, D. C., SÁNCHEZ, F. M., GARCÍA, P. V., \& MARTíNEZ, J. R. (2007). Evaluación en e-learning basada en tecnologías de la Web semántica y procesamiento del lenguaje natural. Murcia: Diego Marín.

CASTELLS, P. (2003). La Web Semántica. En C. Bravo \& M. A. Redondo (Eds.), Sistemas interactivos y colaborativos en la Web (pp. 195-212). Castilla La Mancha: Ediciones de la Universidad de Castilla La Mancha.

CORCHO, O., LÓPEZ, M. F., \& PÉREZ, A. G. (2003). Methodologies, tools and languages for builing ontologies. Where is their meeting point?. Data and Knowledge Engineering, 46(1), 41-64.

ENSEMBLE (2008). Semantic Technologies for the Enhancement of Case-based Learning. LJMU and City University and University of Cambridge. Consultado el 3 de Marzo de 2011 desde http://www.ensemble.ac.uk.

FUA (2011). Free University Amsterdam. FUA. Consultado el 2 de Abril de 2011 desde http://www.vu.nl/nl/index.asp.

FALBO, R. A., RUY, F. B., PEZZIN, J., \& MORO, R. D. (2004). Ontologias e ambientes de desenvolvimiento de software semànticos. En IV Jornadas Iberoamericanas de Ingeniería. Documento electrónico, http://ia.ucpel.tche.br/ Ipalazzo/Aulas/IWS/m09/Recursos/OntoADSS.pdf. [Consultado el 24 de Noviembre de 2010].

GHIA (2010). Grupo de Herramientas Interactivas Avanzadas. Universidad Autónoma de Madrid, UAM. Consultado el 1 de Abril de 2011 desde http://astreo.ii.uam.es/ ghia/.

HERMAN, I. (2010). Introduction to Semantic Web. En 2010 Semantic Technology Conference. Documento electrónico, http://www.w3.org/2010/Talks/0622-SemTechIH/Tutorial.pdf. [Consultado el 12 de Marzo de 2011].

HUYNH, D. F., KARGER, D. R., \& MILLER, R. C. (2008). Exhibit: Lightweight structured data publishing. . Documento electrónico, http://people.csail.mit.edu/dfhuynh/research/papers/www2007-exhibit.pdf [Consultado el 11 de Enero de 2011]

IBRAHIM, B. \& FRANKLIN, S. (1995). Advanced educational uses of the World Wide Web. Computer Networks and ISDN Systems, 27(6), 871--877.

LLII (2010). Learning Lab, Institut fur Informationssysteme. Hannover University. Consultado el 12 de Marzo de 2011 desde http://www.kbs.uni-hannover.de.

MIT (2008). Simile. MIT. Consultado el 10 de Marzo de 2010 desde http://simile.mit.edu/wiki/SIMILE:About.

MAETDCHE, A. D. (2002). Ontology learning for the Semantic Web. The Netherlands: Kluwer Academic Publishers Group.

MILES, A. \& BECHHOFER, S. (2009). Simple Knowledge Organization Classification specification. . W3C. Consultado el 12 de Marzo de 2011 desde http://www.w3.org/TR/2009/REC-skos-reference-20090818/. 
SCIL (2011). Stanford Center for Innovations in Learning. Stanford University. Consultado el 12 de Marzo de 2011 desde http://scil.stanford.edu.

TIROPANIS, T., DAVIS, H., MILLARD, D., \& WEAL, M. (2009). Semantic Technologies for Learning and Teaching in the Web 2.0 era, a survey. En WebSci09: Society On-Line, Grecia, 2009 (pp. 18-20). Documento electrónico,

http://eprints.ecs.soton.ac.uk/17106/1/Websci09-tiropanis.pdf. [Consultado el 25 de Noviembre de 2010].

TSCHOLL, M., TRACY, F., \& CARMICHAEL, P. (2009). Case Methods, Pedagogical Innovation and Semantic Technologies. En 1st International Workshop on Semantic Web Applications for Learning and Teaching Support in Higher Education (SemHE'09). Documento electrónico, http://eprints.ecs.soton.ac.uk/18050/1/semhe09_submission_3.pdf. [Consultado el 15 de Diciembre de 2010].

UNIVERSITY, K. (). KARLSRUHE-UNIVERSITY. Consultado el 12 de Marzo de 2011 desde http://www.uni-karlsruhe.de.

O4E (2009). SWEL: Ontologies and Social Semantic Web for Education. Winston-Salem State University and University of Pittsburgh and Saint-Petersburg University. Consultado el 2 de Abril de 2011 desde http://o4e.iiscs.wssu.edu/drupal/.

Para citar este artículo:

SÁNCHEZ, M. M.; PRENDES, M. P.; MARTínEZ, F.; CARMICHAEL, P.; MARTíNEZ, A. (2011) «Experiencias de incorporación de aplicaciones semánticas a la educación» [artículo en línea]. EDUTEC, Revista Electrónica de Tecnología Educativa. Núm. 36 / Junio 2011. [Fecha de consulta: dd/mm/aa].

http://edutec.rediris.es/revelec2/revelec36

ISSN 1135-9250. 